\title{
Implementación de un laboratorio de habilidades clínicas centralizado en la Facultad de Medicina de la Universitat de Barcelona. Cuatro años de experiencia
}

\author{
A. Mazarro, C. Gomar-Sancho, J. Palés-Argullós
}

Introducción. La Facultad de Medicina de la Universitat de Barcelona definió en su momento las competencias a adquirir por sus estudiantes y evaluó dicha adquisición, apreciándose un déficit en el ámbito de las habilidades clínicas y de los procedimientos técnicos. Para solventar estos déficit, la facultad decidió implementar un laboratorio de habilidades centralizado. Materiales y métodos. Describimos las diferentes fases del proceso de implementación y el desarrollo de un curso optativo sobre habilidades clínicas para adquirir experiencia en la gestión de dicho centro y dar a conocer el recurso entre los profesores de la facultad. Asimismo, hemos investigado el grado de aceptación de este recurso por los alumnos y profesores utilizando diversos cuestionarios. Resultados. En estos cuatro años de experiencia, aproximadamente 1.000 estudiantes cada año, de grado y de posgrado, han recibido docencia en el laboratorio. Los estudiantes consideran el laboratorio como un instrumento excelente para incrementar su competencia individual en habilidades clínicas y que deben extenderse sus prestaciones a todos los estudiantes. Los profesores consideran el laboratorio como un instrumento muy útil para mejorar la enseñanza clínica. Conclusiones. Consideramos que la experiencia ha sido muy positiva y puede ser útil para el proceso de cambio curricular actualmente en curso en la facultad. La experiencia, además, podría servir de modelo para la implementación de laboratorios de habilidades en otras facultades. Finalmente, la Facultad de Medicina debe incrementar su inversión para mejorar los recursos y la accesibilidad del laboratorio a todos los estudiantes y profesores.

Palabras clave. Formación clínica en el grado. Habilidades clínicas. Laboratorio de habilidades clínicas. Simulación.

\section{Implementation of a centralized clinical skills laboratory at the Medical School of the University of Barcelona. Four years of experience}

Introduction. Medical School at the University of Barcelona defined learning outcomes and evaluated its acquisition by the students. Some lacks in several clinical skills and procedures have been detected. To improve clinical skills learning, medical school decided to implement a centralized skills centre. Materials and methods. We describe the different steps of the process of implementation of this centre and the development of an elective course on clinical skills and procedures, in order to acquire experience in the management of this center and to show to the teachers the usefulness of these facilities. Finally, we investigated the level of acceptance of this tool by teachers and students by using different kinds of questionnaires. Results. In the last four years about 1000 medical students per year (undergraduate and postgraduate) have been trained in the laboratory. Students consider that the center is an excellent tool to increase their individual competence in clinical skills and it is necessary to extend this tool to all students. For teachers, the center is a very useful tool to improve clinical training. Conclusions. Our experience has been positive and very useful in the curriculum change process carried out at present in our medical school. The experience could serve as a model for the implementation of skills centers in other Spanish Medical Schools. Medical School must increase the investment in order to improve the facilities and the accessibility of the center to all students and teachers.

Key words. Clinical skills. Simulation. Skills centre. Undergraduate clinical training.
Departamento de Ciencias Fisiológicas I. Facultad de Medicina. Universitat de Barcelona. Barcelona, España.

Correspondencia Dr. Jorge Palés. Departamento de Ciencias Fisiológicas I. Facultad de Medicina. Universitat de Barcelona. Casanova, 143. E-08036 Barcelona.

Fax +34 934035295

E-mail

jpales@ub.edu

Agradecimientos A todos los miembros del Grup Consolidat d'Innovació Docent 2006GIDC-UB/39 de la Universitat de Barcelona, $y$ a los profesores $y$ alumnos becarios e internos que han participado en la experiencia.

Trabajo realizado en parte gracias a l'Ajut per a la Millora de la Qualitat Docent MQD20060006 de la Generalitat de Catalunya y a los fondos recibidos por la concesión de la Distinción Jaume Vicens Vives otorgada a la Facultad de Medicina de la Universitat de Barcelona por la implantación de un laboratorio de habilidades. 


\section{Introducción}

La reforma de los estudios universitarios en el contexto del proceso de Bolonia hace hincapié, entre otros aspectos, en la necesidad de formar a nuestros futuros profesionales en competencias tanto transversales como específicas. En este contexto, diversas instancias, y entre ellas nuestra Facultad de Medicina, han definido tales competencias y en algunos casos se ha evaluado en qué medida dichas competencias son adquiridas actualmente por nuestros estudiantes $[1,2]$. Uno de los ámbitos en los que nuestros estudiantes presentan déficit importantes es el de las habilidades clínicas y los procedimientos clínicos básicos. Estos defectos pueden explicarse por varios motivos, entre ellos la falta de tiempo, durante las estancias clínicas de nuestros estudiantes, para aprender y realizar de forma repetida las diferentes habilidades, la imposibilidad de repetir tantas veces como se quiera una práctica determinada -ya que no se pueden causar molestias a los enfermos, por la falta de capacidad técnica del estudiante o por la posibilidad de cometer errores, con el consiguiente peligro para el enfermo- $y$, finalmente, la existencia de una gran presión de la actividad asistencial que hace muy difícil una atención y supervisión adecuadas de la actuación de los estudiantes por parte de los profesores.

La insuficiente o incompleta adquisición de habilidades clínicas ha sido hasta ahora un hecho común entre los graduados de las facultades de Medicina españolas [3]. Adquirir estas habilidades comporta tiempo, paciencia y práctica. La importancia de asegurar la adquisición de las habilidades clínicas al mismo nivel que los conocimientos y las actitudes han llevado a la proliferación de nuevos entornos de aprendizaje, conocidos como laboratorios de habilidades, que han venido a solucionar, en gran parte, estos problemas.

El primer laboratorio de habilidades estructurado se estableció formalmente en la Facultad de Medicina de la Universidad de Maastricht, en los Países Bajos, en 1976 [4]. Dicho laboratorio facilita entrenamiento en cuatro áreas bien definidas: habilidades de exploración física, habilidades terapéuticas, habilidades de laboratorio y habilidades de comunicación. A partir de aquí se produce una importante proliferación de estos laboratorios en el ámbito mundial, en el contex- to de una facultad de medicina o de un hospital, siendo una constante en la práctica totalidad de facultades de Medicina de Estados Unidos, Canadá, Reino Unido y otros países europeos avanzados. Este crecimiento se debe a varias razones:

- El impresionante desarrollo en los últimos tiempos de diferentes instrumentos en el campo de las simulaciones.

- El progresivo énfasis en la necesidad de que los estudiantes adquieran las habilidades básicas y la capacidad del razonamiento clínico, y fomentar su aprendizaje autónomo.

- Los cambios en el modelo asistencial sanitario.

\section{Desarrollo de instrumentos en \\ el campo de las simulaciones [5-9]}

En la actualidad disponemos en el campo de las simulaciones de diferentes instrumentos:

- Simuladores que permiten un amplísimo abanico de prestaciones. Los maniquíes utilizados pueden ser sencillamente mecánicos o estar fuertemente robotizados, es decir, maniquíes ligados a sistemas informáticos. Estos últimos aumentan enormemente las posibilidades de aprendizaje al permitir trabajar en múltiples situaciones fisiológicas y patológicas, aunque también son muy costosos desde el punto de vista económico y, sobre todo, precisan personal muy entrenado.

- Simulaciones por ordenador o mediante 'pantalla' ('screen simulation'). Este tipo de simulaciones presenta ventajas educativas respecto al uso de pacientes reales en una considerable cantidad de escenarios o situaciones clínicas. Todos los estudiantes pueden estudiar el mismo caso, es fácil conseguir un feedback en sus niveles de conocimientos y habilidades, el estudiante puede cometer errores sin consecuencias y puede disponerse de patologías de las que no siempre resulta posible disponer en la realidad. Los programas pueden construirse con el elemento temporal incorporado, con el fin de dar información al estudiante de las consecuencias de sus decisiones sobre el simulador. Su generalización de uso depende de la disponibilidad de terminales u ordenadores, lo cual no representa un gran problema en nuestro entorno. 
- Enfermos simulados o estandarizados. Los enfermos de este tipo vienen utilizándose desde hace ya más de 30 años, siendo una práctica habitual en muchas facultades de Medicina de todo el mundo. El uso de este tipo de instrumentos facilita el entrenamiento en habilidades de comunicación y la adquisición de competencias fundamentales, como realizar la historia clínica y la exploración física, y aunque ninguna simulación es más real que el paciente 'en vivo', los pacientes simulados se aproximan mucho a la realidad clínica, constituyen un instrumento de transición para enfrentarse a la vida real y resultan muy útiles para obtener un feedback directo de las habilidades desarrolladas por los estudiantes. Los pacientes simulados, al ser capaces de presentar el mismo cuadro de forma repetida, evitan las molestias excesivas a los enfermos reales. Los enfermos simulados alcanzan su máxima utilidad para adquirir aquellas habilidades de tipo genérico, no vinculadas a la especificidad del caso, aunque no son útiles para la enseñanza de las habilidades de procedimientos técnicos. Si bien los costes de selección, entrenamiento y utilización de este tipo de pacientes son elevados, resultan eficientes respecto al coste de tiempo del profesorado necesario para alcanzar resultados similares.

A pesar de todas estas posibilidades, ningún simulador permite por sí solo una enseñanza completa y debe reconocerse que ésta es siempre parcial. La combinación de varios métodos de simulación y, sobre todo, la capacidad del profesor para aproximarlos a la realidad son las claves para obtener el máximo provecho.

\section{Énfasis en la adquisición de las habilidades y del razonamiento clínico y en la necesidad de fomentar el aprendizaje autónomo}

Parece obvio que el hecho de disponer de los laboratorios de habilidades permite que los estudiantes puedan adquirir mejor determinadas habilidades y la capacidad del razonamiento clínico, este último sobre todo con las simulaciones por ordenador. La posibilidad de disponer de laboratorios de habilidades con facilidades de acceso permite también que los estudiantes puedan entrenarse de forma autónoma en una determinada habilidad o procedimiento.

\section{Cambios en el modelo asistencial sanitario}

Actualmente, y al contrario de lo que ocurría hace tiempo en nuestros centros sanitarios, nadie concibe que un paciente ingresado pueda someterse de forma repetida a exploraciones y procedimientos con objeto de entrenar a nuestros estudiantes, ya que esto supone molestias para los pacientes, posibles riesgos para su seguridad al ser realizados por manos inexpertas y, por otra parte, existe la autonomía de los pacientes para oponerse a ello. El entrenamiento en los laboratorios de habilidades permite a los estudiantes realizar su formación en un ámbito no estresante, donde están permitidos los errores y las repeticiones tantas veces como se quiera, con lo que adquieren las bases necesarias para abordar la práctica posterior en pacientes.

\section{Características de los laboratorios de habilidades y consideraciones en su proceso de implementación [5-9]}

Podemos definir los laboratorios de habilidades como entornos educativos donde los estudiantes y los profesionales médicos o sanitarios aprenden diferentes tipos de habilidades en un entorno no estresante, pero próximo a la realidad, independientemente de la disponibilidad de pacientes reales y bajo la supervisión de profesores o de forma autónoma e independiente. Son entornos donde es posible repetir, tantas veces como se desee, la técnica o habilidad concreta, que permiten el error sin consecuencias, su análisis detallado y su corrección, y el control de la complejidad de la situación del aprendizaje, donde el estudiante recibe de forma continua un feedback sobre su actuación y puede ser evaluado de forma objetiva.

En función de los recursos, un laboratorio de este tipo podrá ser más o menos complejo y utilizar alguna o todas las posibilidades tecnológicas antes mencionadas. También, su estructura podrá ser más o menos compleja, desde simples espacios de mayor o menor tamaño -en forma de minilaboratorios donde se enseñen alguna o más habilidades y que con el tiempo podrán adquirir mayor complejidad- hasta zonas que remeden ámbitos clínicos reales, como un box de urgencias o un quirófano con todas sus prestaciones, y donde el abanico de las habilidades y procedimientos a practicar es muy amplio.

De acuerdo con lo expuesto, nuestra facultad decidió en 2005 iniciar el proceso de implemen- 
tación de un laboratorio de habilidades. Con este objetivo comenzó un proceso de inversión con el fin de dotar un espacio adecuado y adquirir un mínimo de material. Asimismo, se planificó un curso piloto optativo con el fin de:

- Adquirir experiencia de un centro de estas características.

- Identificar qué habilidades podían enseñarse en el centro y formar al profesorado en esta metodología docente.

- Conocer la opinión de los estudiantes y de los profesores sobre las diferentes actividades del centro y sobre sus características y funcionamiento.

- Crear cultura entre los profesores y alumnos para el uso de estos entornos y herramientas.

Describimos en este trabajo cómo se ha llevado a cabo la experiencia desarrollada durante los cuatro últimos años, incluyendo los resultados de las opiniones de los profesores y de los estudiantes y presentando datos sobre el funcionamiento del centro durante este tiempo. Planteamos las recomendaciones a tener en cuenta en el uso de dichos entornos y los retos de futuro de acuerdo con nuestra experiencia.

\section{Materiales y métodos}

\section{Organización de novo de un laboratorio de habilidades}

Se desarrollaron las siguientes etapas:

- Adecuación de una área física.

- Inventario de todo el material: durante los últimos años, los diferentes departamentos habían adquirido diverso material, que fue localizado y centralizado en el laboratorio.

- Identificación de las habilidades y procedimientos técnicos a adquirir en el laboratorio [1].

- Adquisición de nuevo material de acuerdo con las habilidades y procedimientos seleccionados

- Entrenamiento de los profesores: 20 profesores de cinco áreas (medicina, cirugía, ginecología, especialidades quirúrgicas y fisiología) se entrenaron en el uso de diferentes simulaciones. Un becario con dedicación de 20 horas por semana y otros cuatro estudiantes que se be-
Tabla I. Talleres desarrollados en el laboratorio durante el curso optativo.

- Exploración otorrinolaringológica

- Sondaje uretral masculino y femenino y tacto rectal

- Manejo de la vía aérea

- Reanimación cardiopulmonar básica y avanzada

- Oxigenoterapia

- Exploración ginecológica y mamaria

- Suturas y drenajes

- Auscultación de tórax

- Vendajes y yesos

- Manejo del enfermo politraumático

- Vías de administración de fármacos

- Comunicación de malas noticias al enfermo crítico

neficiaban de créditos de libre configuración curricular preparaban los diferentes escenarios se acuerdo con las instrucciones de los profesores correspondientes. En el último curso se ha incorporado al laboratorio una persona en calidad de técnico.

\section{Organización de un curso/asignatura piloto optativo}

\section{Estructura del curso}

El curso denominado 'Técnicas y procedimientos clínicos esenciales' se estructuró en 12 talleres a cargo de uno o dos profesores de las asignaturas a las cuales correspondían las habilidades programadas (Tabla I). Para cada taller se elaboró una guía de estudio.

\section{Desarrollo de cada taller}

Cada taller se desarrollaba durante dos horas. Se iniciaba con una breve introducción utilizando material audiovisual. Durante ese tiempo, los estudiantes practicaban las diferentes habilidades bajo la supervisión del profesor y finalmente eran evaluados. Al final de cada taller, los estudiantes disponían de tiempo libre para practicar de forma autónoma el respectivo taller.

\section{Distribución de los estudiantes}

El curso se ofreció a estudiantes de cuarto a sexto año (en total, 720). Se admitieron cada año 40- 
Tabla II. Puntuaciones medias obtenidas por todos los estudiantes en cada ítem, evaluado de 0 a 10 , globalmente para los 12 talleres.

\begin{tabular}{lc}
\hline Interés demostrado durante el taller & 9,60 \\
\hline Actitud demostrada durante el taller & 9,66 \\
\hline $\begin{array}{l}\text { Realización de la habilidad y la técnica } \\
\text { Capacidad para enseñar la } \\
\text { habilidad a otros estudiantes }\end{array}$ & 9,10 \\
\hline
\end{tabular}

46 estudiantes distribuidos en grupos de diez. El procedimiento de selección fue el normalmente establecido por la facultad para todas las asignaturas optativas.

\section{Evaluación de los estudiantes}

Los estudiantes fueron evaluados por observación directa utilizando listas de comprobación específicas. Los ítems de dichas listas se muestran en la tabla II y se puntuaban de 0 a 10. Además, se consideraba condición sine qua non para superar el curso la asistencia a más de un $80 \%$ de los talleres.

\section{Opinión de los estudiantes}

sobre el desarrollo del curso

Cada taller y el desarrollo global del curso fueron evaluados por los estudiantes de acuerdo con un cuestionario usando una escala de tipo Likert (Tabla III), desde 1 (máximo desacuerdo) a 5 (máximo acuerdo).

\section{Opinión de los estudiantes sobre las}

prestaciones y las características del laboratorio Al final de cada curso, se plantearon diferentes cuestionarios utilizando una escala de tipo Likert. Los ítems (Tabla IV) se valoraban desde 1 (máximo desacuerdo) a 5 (máximo acuerdo).

\section{Aceptación del curso por los estudiantes}

Debido a razones técnicas, el curso no estaba abierto a todos los estudiantes de la facultad, que habían de realizar una preinscripción. Al final del segundo año de experiencia, una vez los estudiantes habían realizado su preinscripción, inves- tigamos el grado de aceptación de los estudiantes de la facultad, de acuerdo con los datos de dicha preinscripción. Como los diferentes cursos optativos tienen prerrequisitos distintos y no se ofrecen al mismo número de estudiantes, no pareció adecuado comparar sólo el número absoluto de peticiones para el curso y consideramos más pertinente comparar el porcentaje de alumnos que solicitaban una plaza en nuestro curso sobre el total de posibles candidatos que podían solicitarlo.

\section{Uso del laboratorio para otras actividades docentes}

Dada la importancia de un centro como el nuestro, tanto para la educación graduada como posgraduada y la formación médica continua [10], ofrecimos también las prestaciones del laboratorio para actividades formativas de estudiantes de Medicina en diferentes asignaturas y de posgraduados y médicos residentes.

\section{Resultados}

\section{Organización del laboratorio}

\section{Adecuación de una área física}

El área del laboratorio, de aproximadamente $150 \mathrm{~m}^{2}$, se dividió en cinco módulos que podían usarse de forma independiente o conjuntamente en diferentes combinaciones, disponiendo todos ellos de medios audiovisuales y conexión a red. Uno de los módulos cuadrados se amuebló simulando un box de emergencias hospitalarias.

\section{Identificación de las habilidades clínicas y los procedimientos prácticos a enseñar en el laboratorio}

De acuerdo con estudios previos y las prestaciones del laboratorio, se decidió enseñar aquellas habilidades que se muestran en la tabla I.

\section{Organización del curso optativo}

\section{Talleres}

Se muestran en la tabla I.

\section{Guía de estudio}

Para cada taller se elaboró una guía o manual de estudio que contenía los objetivos específicos, el 
Tabla III. Puntuaciones medias otorgadas por los estudiantes en los diferentes ítems, en todos los talleres, de 0 a 5 .

\begin{tabular}{lc}
\hline Interés del taller & 4,70 \\
\hline Utilidad del taller & 4,65 \\
\hline Después del taller me siento capaz de realizar la habilidad en situación simulada & 4,60 \\
\hline Después del taller soy capaz de realizar la habilidad en situación real & 4,20 \\
\hline Después del taller soy capaz de enseñarla a otros estudiantes & 4,35 \\
\hline Ver a otros estudiantes realizar la técnica me ayuda a aprender & 4,55 \\
\hline El profesor me ha enseñado correctamente & 4,80 \\
\hline Se han alcanzado los objetivos del curso & 4,50 \\
\hline El curso resuelve un déficit importante del plan de estudios & 4,75 \\
\hline El curso debe incluirse en el currículo troncal & 4,60 \\
\hline Recomendaría el curso a otros estudiantes & 4,75 \\
\hline
\end{tabular}

material necesario, las bases teóricas de la técnica, la metodología para la realización de la habilidad o técnica, los principales errores o complicaciones de la técnica, y el método de evaluación.

\section{Desarrollo del curso}

El curso se desarrollaba durante cuatro meses. Además de la dedicación de dos horas a cada taller, el estudiante disponía de tiempo libre para continuar su aprendizaje de forma autónoma.

\section{Evaluación de los estudiantes}

Los resultados se muestran en la tabla II. Sólo dos estudiantes en el primer año, tres durante el segundo y el tercero, y dos en el cuarto no superaron el curso por no asistir al $80 \%$ de los talleres programados. La media de las puntuaciones de los estudiantes en todos los ítems fue de 8,6 sobre 10 .

Opinión de los estudiantes sobre el desarrollo de los diferentes talleres y del curso en su globalidad Los resultados se muestran en la tabla III, siendo muy positivos en todos los ítems.
Opinión de los estudiantes y profesores sobre las prestaciones del laboratorio Las opiniones se muestran en la tabla IV, desde 1 (máximo desacuerdo) hasta 5 (máximo acuerdo).

\section{Análisis de la aceptación del curso}

Para este análisis hemos utilizado datos de la segunda y tercera edición del curso (cursos académicos 2006-2007 y 2007-2008), pero no los de la primera edición. Consideramos que el número de peticiones de la primera edición no puede tenerse en cuenta porque los estudiantes no conocían exactamente las características del curso hasta que éste no se desarrollara al menos en una edición.

El número total de preinscripciones para las diferentes asignaturas optativas de nuestra facultad varió desde 1 (mínimo) a 261 (máximo) en la segunda edición del curso y desde 0 a 212 en la tercera edición. Nuestro curso recibió 135 peticiones de preinscripción en la segunda edición y 158 en la tercera. De acuerdo con estas cifras, nuestro curso fue el cuarto con más peticiones en la segunda edición y el tercero en la siguiente. 
Teniendo en cuenta el porcentaje de preinscripciones respecto al total de posibles alumnos que podían solicitar el curso, nuestro curso fue el segundo con mayor grado de interés $(28,3 \%$ en un intervalo entre $0,1-36,3 \%$ ) en la segunda edición y el primero en la tercera edición $(32,9 \%$ en un intervalo entre $0-32,9 \%$ ).

\section{Uso del laboratorio de habilidades para otras actividades}

En el laboratorio de habilidades se desarrollaron también actividades docentes correspondientes a distintas asignaturas obligatorias de Medicina y para formación posgraduada. Para los organizadores de cursos de formación posgraduada, los centros de habilidades clínicas aparecen como entornos muy adecuados para la formación de residentes y para la formación continuada de médicos y otros profesionales de las ciencias de la salud [10]. En el conjunto de los dos primeros años, el centro acogió 22 cursos para posgraduados, con casi 1.000 alumnos. Estos cursos requirieron la utilización de gran cantidad de material y una gran ocupación del laboratorio en términos de tiempo. La experiencia fue considerada muy positiva tanto por los organizadores como por los alumnos.

\section{Discusión}

La Facultad de Medicina de la Universitat de Barcelona definió las competencias a adquirir por sus graduados y el grado de adquisición de éstas, detectándose diferentes déficit $[1,2]$. El déficit en la adquisición de las habilidades clínicas es un hecho común en las facultades de Medicina. En los últimos años, el desarrollo de las simulaciones en educación médica y la implementación de los denominados centros o laboratorios de habilidades se han considerado como una de las posibilidades de superar este problema [5-9,11]. Además, la necesidad de preservar la seguridad de los pacientes durante el proceso de aprendizaje ha determinado que la educación médica basada en el uso de las simulaciones se haya convertido en un imperativo ético. Preservar la seguridad y la intimidad de los pacientes puede entrar en conflicto con el aprendizaje de los estudiantes en cualquier momento del proceso educativo de los profesionales y las simulaciones pueden minimizar este conflicto ético
[12] Por otra parte, la cada vez más frecuente presión asistencial en el entorno clínico dificulta la dedicación del profesor clínico y la disponibilidad del paciente y de la estructura sanitaria.

Para mejorar la enseñanza de las habilidades clínicas, nuestra facultad implementó hace cuatro años un laboratorio centralizado de habilidades. La implementación de un centro como éste implica una importante inversión económica para adecuar un espacio físico conveniente con diferentes prestaciones. Aun reconociendo que los aspectos financieros son sin duda importantes en este proceso, existen otros aspectos igualmente importantes. Es necesario tener un proyecto educativo bien diseñado para desarrollarlo en el centro y convencer a los profesores de la necesidad del uso de las simulaciones y de sacar partido de las facilidades que les puede ofrecer un centro de este tipo. Debe convencerse a los departamentos para que consideren en su presupuesto la adquisición de este tipo de material y convenzan a sus profesores para que se impliquen. La experiencia demuestra que cuando los distintos profesores conocen el proyecto, lo hacen crecer exponencialmente con ideas y sugerencias de material para simulaciones.

Carecíamos de experiencia en la gestión de un laboratorio de este tipo y los profesores tenían pocos conocimientos sobre el uso de las simulaciones como instrumentos docentes. Por estas razones decidimos desarrollar un curso optativo en el cual los estudiantes pudiesen adquirir las habilidades y procedimientos clínicos básicos y los profesores pudiesen conocer dichos recursos. Los contenidos del curso se establecieron de acuerdo con los déficit observados y descritos anteriormente [1]. Diferentes profesores de varios departamentos fueron invitados a participar en el curso con el fin de que apreciasen la utilidad del laboratorio. Al final de cada taller y al final del curso, se solicitó a los estudiantes que evaluasen las características del laboratorio. Dicha opinión fue muy positiva en todos los aspectos. Consideraron que los diferentes talleres fueron muy interesantes y útiles y se consideraban capaces de realizar el procedimiento o la habilidad en una situación real. La opinión más relevante es que consideraban que el curso venía a llenar una importante laguna de su currículo y debía incorporarse como una actividad troncal. Asimismo, los estudiantes manifestaban que aprendían mucho observando cómo sus compañeros realizaban la técnica y que 
Tabla IV. Opinión de los profesores y estudiantes sobre las características del laboratorio.

\begin{tabular}{lcc}
\hline & Profesores & Estudiantes \\
\hline El laboratorio tiene una estructura adecuada & 4,24 & 3,95 \\
\hline Los recursos materiales son adecuados & 4,20 & 4,00 \\
\hline El personal de soporte es adecuado & 4,60 & 4,35 \\
\hline Las tareas del personal de soporte se han desarrollado adecuadamente & 4,75 & 4,40 \\
\hline El laboratorio es una herramienta para mejorar la adquisición de competencias & 4,45 & 4,55 \\
\hline El laboratorio es una herramienta para mejorar el aprendizaje autónomo & 4,20 & 4,40 \\
\hline Debe asegurarse el libre acceso al laboratorio & 4,35 & 4,40 \\
\hline
\end{tabular}

ello puede incrementar la eficiencia del aprendizaje en el laboratorio. Por otra parte, se preguntó a los estudiantes y profesores su opinión sobre las prestaciones del laboratorio En general, la opinión vuelve a ser muy positiva y no se aprecian diferencias relevantes entre ambos grupos, aunque la opinión de los estudiantes es ligeramente más crítica en lo que se refiere a aspectos estructurales. Los estudiantes consideran que el laboratorio es una herramienta fundamental para mejorar el aprendizaje autónomo y que resulta necesario asegurar el libre acceso en un horario amplio. Los profesores están de acuerdo con estas opiniones, aunque muestran alguna reticencia con respecto al aprendizaje autónomo. Quizás estas diferencias pueden explicarse por su concepción más tradicional de la enseñanza y del aprendizaje.

Consideramos que esta enseñanza debe organizarse de modo que no cueste un esfuerzo adicional al profesor. La clave es que éste encuentre el taller organizado según sus preferencias a la hora de iniciar la clase y que puede marcharse de ésta al final de su docencia dejando la tarea de revisión del material en manos técnicas expertas de los encargados de material del laboratorio.

Otra principal conclusión de nuestra experiencia es que el curso fue uno de los más atractivos de todas las materias optativas que se ofrecían a los estudiantes de nuestra facultad. Los resultados obtenidos después de cuatro años muestran un alto nivel de aceptación. De acuerdo con ello, pensamos que también hemos alcanzado otro objetivo: ofrecer una forma práctica de enseñanza y aprendizaje de las habilidades clínicas básicas con una excelente aceptación entre los estudiantes. Estos resultados coinciden con las sugerencias de la IFMSA (International Federation of Medical Students' Associations) y de la EMSA (European Medical Students' Association) [13] y con otras experiencias previas [14]. El alto nivel de aceptación de estudiantes y profesores representa para nosotros un fuerte estímulo para continuar con este proyecto. Su feedback debe interpretarse como un mensaje de apoyo para extender esta herramienta a todos los estudiantes de la facultad.

De cara al futuro se presentan algunos retos. En primer lugar, es necesario incrementar el espacio físico a corto plazo porque nuestra experiencia demuestra que se requiere un espacio mayor y más funcional. Además, si es necesario extender el uso del laboratorio a todos nuestros estudiantes en un horario más amplio, se deben incrementar los recursos humanos, especialmente técnicos y personal administrativo, para asegurar el mantenimiento del material. Otro reto esencial es completar las prestaciones del centro, sobre todo en aquellos campos todavía no desarrollados como las habilidades de comunicación y el uso de pacientes simulados.

Actualmente, un objetivo es hacer que la mayor parte de las asignaturas clínicas y algunas básicas 
enseñen, mediante técnicas de simulación, todo aquello que es posible en su materia e integren esta parte de su docencia en el entorno del laboratorio. Creemos que es totalmente necesario incorporar la enseñanza de las habilidades y procedimientos clínicos básicos en el nuevo currículo, integrándolo en las diferentes asignaturas troncales u obligatorias y considerándola un recurso docente complementario pero imprescindible. Debemos asegurarnos de que nuestros estudiantes puedan aprender las diferentes habilidades antes de cursar su estancia clínica en los hospitales y centros de salud, y que puedan utilizar las prestaciones del laboratorio en cualquier momento para reforzar la adquisición de dichas habilidades.

En general, consideramos que nuestra experiencia ha sido muy positiva y estamos seguros de que permitirá mejorar el aprendizaje de las habilidades clínicas y mejorar la gestión del centro. Para confirmar nuestra percepción hemos iniciado un estudio comparativo de los resultados obtenidos en la evaluación clínica objetiva estructurada (ECOE) que nuestra facultad viene realizando los últimos años, por estudiantes que han seguido nuestro curso y aquellos que no han tenido dicha oportunidad. Los resultados, aunque muy preliminares y con un número todavía pequeño de estudiantes evaluados -lo cual hace que se deban considerar con mucha cautela-, parecen indicar que el rendimiento de los estudiantes que han realizado nuestro curso es mejor que el de los estudiantes que no lo hicieron (puntuación media de los estudiantes que siguieron el curso, $59 \%$; puntuación media del resto de estudiantes, $50 \%$; media de todos los estudiantes evaluados por ECOE, 52\%).

Para acabar, después de cuatro años de experiencia podríamos plantear algunas recomendaciones:

- Actualmente, nuestras facultades de Medicina han de ser conscientes de la necesidad de implementar, dentro de sus posibilidades, estos laboratorios de habilidades. En palabras de Amitai Ziv [12], 'la educación médica basada en simulaciones es hoy día un imperativo ético. Preservar la seguridad de los pacientes entra en conflicto con el aprendizaje de los futuros profesionales en algún momento del proceso educativo. Las simulaciones pueden minimizar este conflicto ético'. Sin embargo, el uso de las simulaciones, para ser racional, debe basarse en un programa docente perfectamente establecido y el entorno educativo de un laboratorio de habilidades es la estructura que permite sin duda dicho uso racional.

- La enseñanza de las habilidades en entornos de este tipo ha de estar integrada perfectamente en el currículo y en relación con la actividad clínica del estudiante. Lo que se enseña debe ser relevante en el contexto. Se debe planificar la enseñanza de las diferentes habilidades de forma integrada con la enseñanza teórica y práctica que ha de recibir el alumno y el material que se adquiera debe estar en perfecta consonancia con los objetivos de aprendizaje que se deseen alcanzar.

- Los laboratorios de habilidades no pueden ser centros aislados del entorno clínico real y se debe ser consciente de sus limitaciones y de las limitaciones de la tecnología. El manejarse correctamente con el simulador no es igual a competencia clínica.

- Por el elevado costo que representa un laboratorio de este tipo en una facultad, es necesaria una adecuada conexión con el centro o los centros hospitalarios relacionados. Los laboratorios de las facultades y los centros sanitarios deben compartir los recursos, tanto materiales como humanos, para rentabilizarlos, y éstos deben utilizar las prestaciones de aquéllos para la formación continua de sus profesionales.

- Todo el profesorado, y en especial los clínicos, deben involucrarse en las actividades de los laboratorios de habilidades y en su planificación y conocer todo lo que en él se enseña. Deben ser conscientes de facilitar a sus estudiantes todo el soporte necesario cuando éstos desarrollen en la realidad las diferentes habilidades aprendidas en el laboratorio.

- El laboratorio de habilidades debe disponer de personal que asegure su mantenimiento y el de sus recursos. Debe existir personal administrativo que asegure un correcto funcionamiento del mismo y un libre acceso en amplios períodos diarios a todos los posibles usuarios y personal técnico especializado en el manejo y mantenimiento de los recursos. La complejidad tecnológica de determinados recursos exige la existencia de este personal para rentabilizar las prestaciones que estos recursos poseen.

- Los usuarios de los laboratorios de habilidades deben ser conscientes de que, aunque trabajan en un entorno de simulación, han de actuar de 
la misma manera como lo harían en la realidad. El material de simulación no puede considerarse como un mero juguete y en su manejo han de observarse las mismas condiciones de uso y seguridad que en la realidad.

\section{Bibliografía}

1. Palés J. Defining the learning outcomes of graduates from the Medical School at the University of Barcelona (Catalonia, Spain). Med Teach 2004; 26: 239-43.

2. Palés J, Gual A, Gomar C, Estrach MT. Acquisition of learning outcomes by students from the Medical School of the University of Barcelona (Catalonia, Spain): a student survey. Med Teach 2008; 30: 693-8.

3. Palés J, Gual A, Medical education in Spain: current status and new challenges. Med Teach 2008; 30: 365-9.

4. Bouhuijs PA, Schmidt HG, Snow RE, Wijnen WHFW. The Rijksuniversiteit Limburg, Maastricht, Netherlands: development of medical education. Public Health Papers. Geneva: WHO; 1978. p. 133-51.
5. Ledingham I, Harden RM. Twelve tips for setting up a clinical skills teaching facility. Med Teach 1998; 20: 503-7.

6. Stark P, Fortune F. Teaching clinical skills in developing countries: are clinical skills centres the answer? Educ Health 2003; 16: 298-306.

7. Dent J. Current trends and future implications in the developing role of clinical skills centres. Med Teach 2001; 23: 483-9.

8. Ker JS. Clinical skills center teaching. In Dent J, Harden R, eds. Practical guide for medical teachers. Edinburgh: Churchill-Livingstone/Elsevier; 2009. p. 86-95.

9. Dacre J, Nicol M, Holroyd D, Ingram D. The development of a clinical skills centre. J R Coll Physicians Lond 1996; 30: 318-24.

10. Bligh J. Clinical skills unit. Postgrad Med J 1995; 71: 730-2.

11. Hao J, Estrada J, Tropez-Sims S. The Clinical Skills Laboratory: a cost effective venue for teaching clinical skills to third year medical students. Acad Med 2002; 77: 152.

12. Ziv A, Wolpe P, Small S, Glick S, Simulation-based medical education -an ethical imperative. Acad Med 2003; 78: 783-8.

13. Hilgers J, De Roos P, Rigby E. European core curriculum. The student's perspective. Med Teach 2006; 29: 270-5.

14. Weller J. Simulation in undergraduate medical education: bridging the gap between theory and practice. Med Educ 2004; 38: 32-8. 